\title{
Um modelo de software colaborativo com suporte à troca de informações entre equipes médicas plantonistas
}

\author{
Vinícius Tocantins Marques ${ }^{1,2}$, Cristiano André da Costa ${ }^{1}$, \\ Jorge Luis Victoria Barbosa ${ }^{1}$, Rodrigo da Rosa Righi ${ }^{1}$ \\ ${ }^{1}$ Programa de Pós-Graduação em Computação Aplicada - PIPCA - UNISINOS \\ ${ }^{2}$ Instituto Federal de Educação Ciência e Tecnologia de Roraima - IFRR \\ vinicius.tocantins@ifrr.edu.br, \{cac, jbarbosa,rrrighi\}@unisinos.br
}

\begin{abstract}
The use of applications that standardize information for emergency environments is one of the main tools for managing health teams in this new century. Ubiquitous computing and multilevel task management are important elements for the exchange of these teams, in which the physician can insert and view patient's medical record data in a way that optimizes their work in a high-stress environment. More specifically, the application can correlate data from heterogeneous sources to attain healthcare success, fostering a wide collaboration network. This article proposes the development of a collaborative model, named Doctor Collab, to support the exchange of information among on duty health personnel using task management and situation aware. The model aims at using computing resources-based activity and ubiquitous computing to improve the management of relevant data in the application, as well as the use of Bayesian networks for medical inferences. The evaluation of the Doctor Collab was made using usage scenarios.
\end{abstract}

Resumo. $O$ uso de aplicações que padronizam informações utilizadas em ambientes de emergências é uma das grandes ferramentas de apoio para as equipes de médicos plantonistas neste novo século. A computação ubíqua e o gerenciamento de tarefas em multinível são elementos de evolução para aplicações computacionais durante a troca dessas equipes, em que o médico possa inserir e visualizar dados do prontuário do paciente de uma maneira que otimize seu trabalho em ambiente de grande stress. Mais especificamente, a aplicação que consegue correlacionar dados de fontes heterogêneas em prol do sucesso do atendimento médico, produzindo uma ampla rede de colaboração. Nesse âmbito, o presente trabalho propõe o desenvolvimento de um modelo colaborativo denominado Doctor Collab. $O$ modelo suporta a troca de informações entre equipes médicas plantonistas utilizando gerenciamento de tarefas e ciência de situação. Doctor Collab visa utilizar recursos de computação baseada em atividade e computação ubíqua para melhorar a manipulação de dados relevantes na aplicação, bem como o uso de redes Bayesianas para inferências médicas. A Avalição do Doctor Collab foi feita através de cenários de uso.

\section{Introdução}

Criar soluções que auxiliem médicos plantonista é fundamental para a eficácia médica. O processo de tomada de decisão sempre foi um dos principais pilares 
almejados por instituições que buscam qualidade em seus trabalhos, e na área da saúde não é diferente. Para que isso possa ser possível, estima-se conectar dispositivos a uma grande rede de informações colaborativas fazendo com que os mesmos possam aumentar o potencial de eficiência da aplicação [Musen et al. 2014].

A tecnologia computacional, de modo intrínseco, compreende uma necessidade de se trabalhar de forma colaborativa. Isso se deve em fato à crescente cooperação entre equipe diante da execução de tarefas do dia, fazendo assim que sistemas colaborativos estejam cada vez mais presentes e difundidos [Shelbourn 2006]. Esse tipo de inteligência computacional poderá muitas vezes ser visto em modelos baseados em computação ubíqua. Ele age de forma mais proativa, criando situações em que o auxílio a um ser humano se torne mais transparente ao ponto que interrompê-lo ao mínimo [Weiser 1991]. Nos sistemas ubíquos, essa possibilidade é explorada com a ciência de situação, em que se pode representar as habilidades humanas, seus efeitos, decisões e modos de operação [Endsley 2015]. Essa técnica pode estar presente em sistemas na qual trabalhe com a visão baseada em decisão onde o dinamismo dos contextos é evidente.

Neste âmbito, o objetivo deste trabalho é propor um modelo colaborativo baseado em nuvem computacional móvel [Fernando, N., Loke, S. W., \& Rahayu, W. 2013] que auxilie a troca de informações entre médicos plantonistas, denominado Doctor Collab. O sistema se utilizará de conceito da computação ubíqua, armazenado em uma base ontológica [Noy, N. F., \& McGuinness, D. L. 2001] organizada e visualizada através de dispositivos móveis e/ou vestíveis. O mesmo deverá ter suporte a colaboração bem como gerenciar tarefas entre usuários que podem estar fisicamente distantes.

Como contribuição científica, o modelo proposto emprega inferências através do uso de Redes Bayesianas para dar suporte à colaboração entre as equipes na área de saúde. O modelo possui um conjunto de módulos em que estão distribuídas suas funcionalidades, no intuito de prover um melhor acesso aos dados de prontuário médico. O Doctor Collab tem um arquitetura baseada em nuvem computacional e acessada a partir de dispositivos móveis.

O artigo está organizado da seguinte forma: a seção 2 descreve a arquitetura e funcionalidades do Doctor Collab. A seção 3 apresenta uma Implementação e Avaliação do modelo. A seção 4 discute os trabalhos relacionados. Por fim, a seção 5 apresenta as considerações finais do trabalho.

\section{Modelo Proposto}

O Doctor Collab é um modelo que auxilia a colaboração de atividades de uma emergência médica de forma a facilitar a troca de informações entre médicos plantonistas. Nesse modelo, algumas premissas foram definidas: (i) processamento em nuvem computacional; (ii) suporte a colaboração na camada de aplicação; (iii) organização das informações em base ontológica; (iv) elasticidade, escalabilidade e disponibilidade fornecidas pela arquitetura em nuvem.

A Figura 1 apresenta uma visão geral do Doctor Collab. Nela pode-se observar que tanto a primeira equipe médica quanto a segunda tem acesso aos dados do paciente em uma base de dados em nuvem computacional. Além disso, os profissionais de saúde estão autorizados a contribuírem de forma colaborativa para a evolução do quadro médico do paciente, utilizando padrões pré-estabelecidos e gerenciamento de tarefas, 
objetivando a consciência situacional.

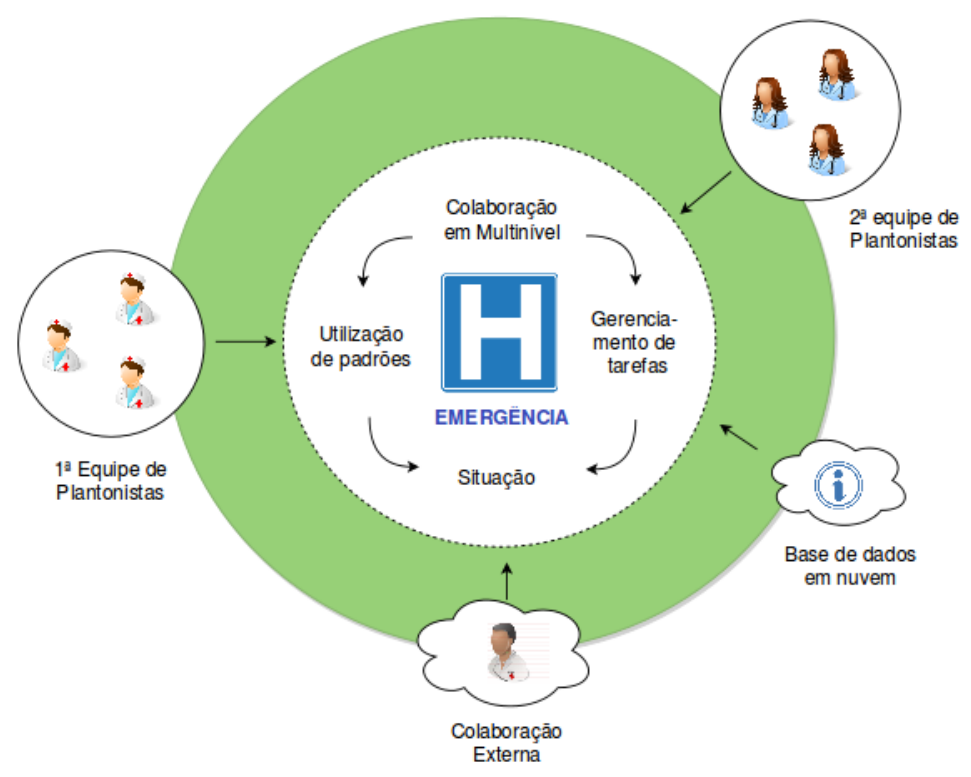

Figura 1 - Visão Ampla do Modelo Doctor Collab

O modelo proposto é inspirado nos conceitos de computação em nuvem móvel [Dinh, H. T. Et al. 2013]. Essa proposta garante o amplo acesso às informações por qualquer pessoa autorizada, em qualquer lugar e a qualquer momento. Além de facilitar a colaboração externa de outros médicos plantonista, bem como atualizar a equipe médica que assumirá o próximo plantão médico. Cria-se assim uma dinâmica mais organizada de colaboração e troca de informações.

No Doctor Collab, o acesso pode ser realizado por dispositivos heterogêneos, garantindo assim uma independência de plataforma e facilitando o acesso dos médicos aos prontuários. Essa entrada no sistema é verificada através de uma interface de controle de acesso. Quando autorizado, o médico poderá interagir com as atividades de cada paciente (conjunto de tarefas), além de seus contextos que influenciarão no melhoramento do processo de tomada de decisão através do uso de ciência de situação.

A plataforma suporta ainda a colaboração através de recursos multimídia, a exemplo de áudios e vídeos, facilitando a comunicação assíncrona. Também são suportadas as comunicações síncronas, a exemplo de videoconferências que poderão ser eventualmente realizadas entre os plantonistas e colaboradores externos.

Esses recursos darão suporte a aplicação de Ciência de Situação, que dentro do modelo que pode ser definido como uma solução que favorece o dinamismo computacional e uma visão baseada em decisões. Ciência de Situação também pode ser representada como uma abstração em alto nível de diversos contextos com o objetivo de aprimorar sistemas em prol das necessidades do ser humano. [LOPES, Joao et al. 2014]

A Figura 2 apresenta a arquitetura detalhada do modelo proposto. Uma das principais características dessa estrutura é o seu paradigma baseado em componentes, utilizando conceitos de reutilização e interação entre cada um. Os componentes presentes nessa arquitetura foram criados no intuito de oferecer um melhor controle entre as informações trafegadas durante a troca de plantões médicos. Seus componentes podem ser detalhados, de forma a otimizar o entendimento da estrutura da arquitetura, são eles: 
SBCUP - VII Simpósio Brasileiro de Computação Ubíqua e Pervasiva

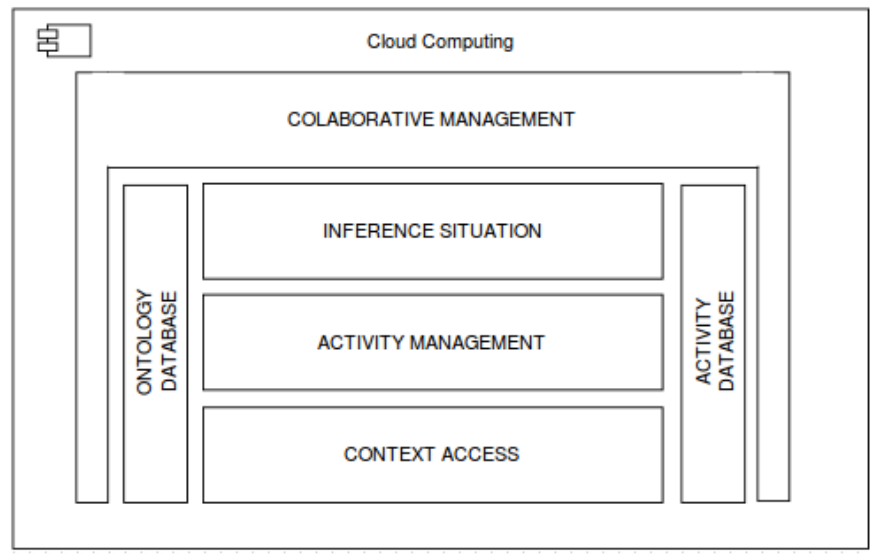

Figura 2 - Modelo detalhado - Servidor Doctor Collab

- Context Access (CA): disponibiliza o acesso aos dados brutos de contextos capturados no ambiente de emergência médica. Essas informações poderão ser obtidas através de sensores;

- Activity Management (AM): responsável pelo gerenciamento da atividade realizada durante um atendimento médico. Uma atividade pode conter um conjunto de tarefas, e esta pode ser uma simplificação de um procedimento documental encontrado em um prontuário médico, podendo conter ali um medicamento utilizado pelo paciente, ou ainda uma recomendação de um médico para o próximo plantonista. Esse componente também dará suporte para que clientes registrados em uma atividade específica, sejam notificados quando tarefas ocorrerem;

- Inference Situation (IS): responsável pela ciência de situação através de inferências na ontologia. O cliente do Doctor Collab terá duas opções, acessar diretamente o conjunto de informações de contexto (dados brutos) ou utilizar esse componente no intuito de obter informações mais precisas, ou ainda ter acesso a informações que só podem ser obtidas através de inferência computacional. As predições serão realizadas através do uso de técnicas de redes Bayesianas e inferências do tipo NP-Hard, onde se apresentam problemas de decisão da soma de subconjuntos, situação comum em casos de escolhas do tipo Sim/Não. Para essas inferências serão utilizadas as ontologias armazenadas no componente Ontology Database. Essas ontologias tem papel fundamental nas inferências uma vez que demostram uma organização do conhecimento em questão, associando o Doctor Collab à realidade. Uma vez descrito a ontologia, a mesma poderá representar regras lógicas para representações de questões não explicitamente representadas.

- Ontology Database (OD): armazena a ontologia utilizada no modelo. Esse componente se faz necessário no intuito de facilitar a exploração dos contextos e situações empregados nas inferências;

- Activity Database (AD): fornece o armazenamento de tarefas e consequentemente atividades realizadas dentro do modelo, ações realizadas no ambiente de uma emergência médica hospitalar;

- Collaborative Management (CM): controla a interação entre as equipes médicas e consequentemente as trocas de informações entre elas. É possível controlar os acessos através da análise das sessões ativas e ainda garantir a troca de 
informações instantâneas por meio de comunicações síncronas e comunicações posteriores através de comunicações assíncronas.

A Figura 3 apresenta uma inferência dentro do modelo Doctor Collab, em que se observa a relação entre o ambiente, a medicação, e possíveis causas do estado de saúde do paciente e as suas probabilidades de ocorrência. Essa informações são úteis para diagnóstico de uma parada cardíaca e ativação de médicos especialista. Com essas informações pode-se calcular a probabilidade de uma medicação, juntamente com causas naturais, ocasionarem uma parada cardíaca em um paciente na emergência médica, por exemplo.

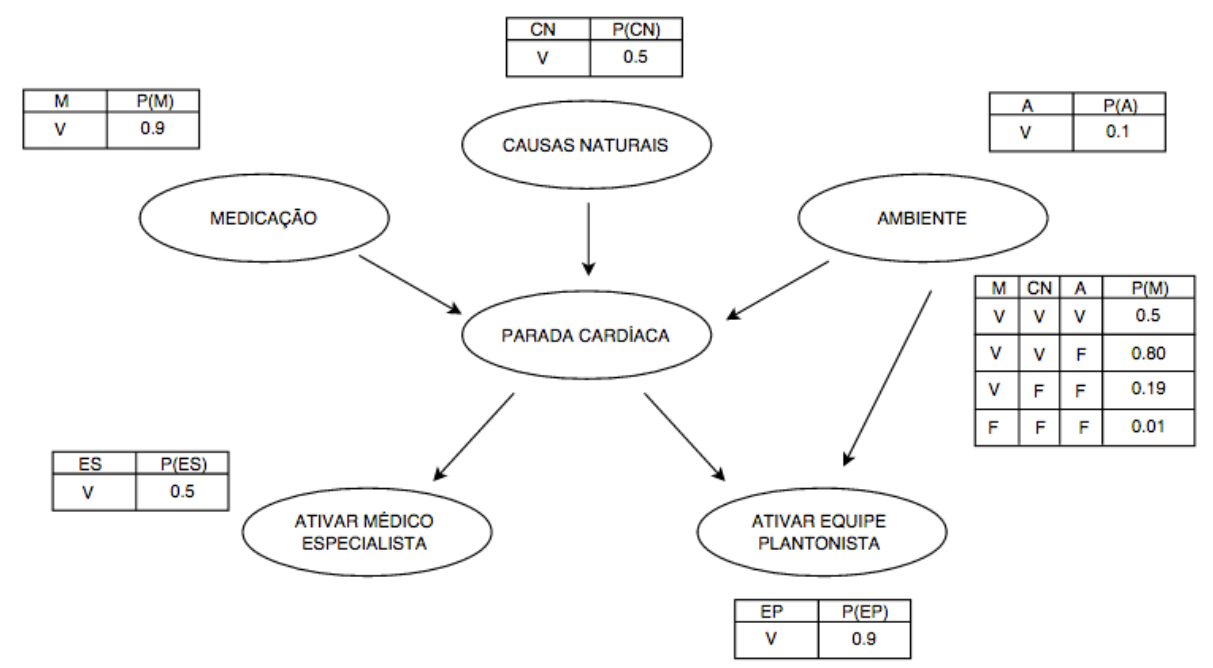

Figura 3 - Rede Bayesiana - Doctor Collab

Para realizar a ciência de situação, foi desenvolvida uma ontologia para o Doctor Collab. Após pesquisa em bases científicas, não foram encontradas ontologias que integrassem ciência de situação, computação baseada em atividades e colaboração para ambiente de emergência médicas. Por isso, optou-se em construir uma nova ontologia utilizando a ferramenta WebProtégé ${ }^{l}$ a partir de estruturas da Ontology Web Language OWL.

Com o uso de uma estrutura ontológica, espera-se responder algumas questões de competência do modelo Doctor Collab: i) Qual a classificação de risco da saúde do paciente conforme seus batimentos cardíacos?, ii) Que tipo de medicação disponível no hospital seria a mais indicada conforme o histórico do paciente a ser atendido?, e iii) Qual médico, mesmo fisicamente longe do hospital, poderia colaborar com a melhora do quadro clínico de um paciente?

A Figura 4 apresenta a ontologia proposta pelo Doctor Collab. As classes macros disponíveis para o modelo são: (i) Acesso: Classe que representa os níveis e permissões de acesso necessários para o controle e monitoramento de acesso, colaboração e troca de informações, (ii) Ativo: Classe que representa todos os ativos de sistemas disponíveis para uso, como dispositivos médicos, informações da equipe, sensoreamento e ainda prontuário, (iii) Colaboração: Classe que representa o estado de colaboração entre médicos do modelo, podendo ser síncrono ou assíncrono, (iv) Risco: Classe que atua na estruturação dos riscos provenientes do comprometimento da

\footnotetext{
${ }^{1}$ Ferramenta para colaboração e desenvolvimento de ontologies obtida em http://webprotege.stanford.edu
} 
integridade, disponibilidade e confidencialidade das informações trafegadas dentro do modelo, e (v) Vulnerabilidade: Classe que atua na verificação de vulnerabilidade de informações do sistema, garantindo o sucesso do tráfego das informações trafegadas dentro do modelo. Vale destacar a existência de subclasses dentro da Ontologia proposta. Elas representam informações mais específicas de cada classe mãe, e proporcionam assim uma melhor estruturação da informação.

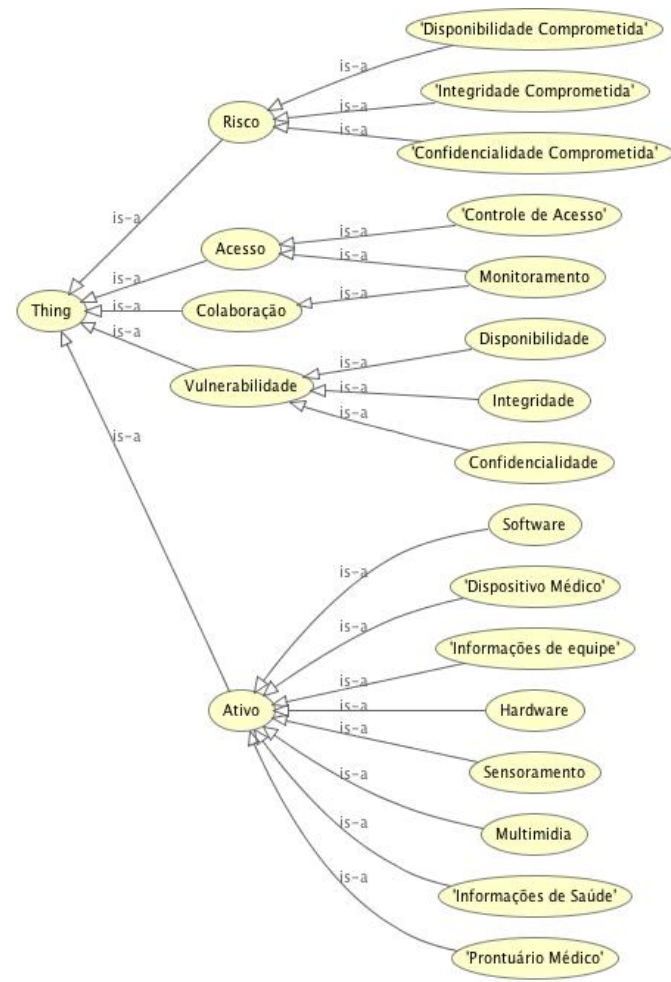

Figura 4 - Ontologia Doctor Collab

\section{Implementação e Avaliação}

Para uma avaliação inicial são utilizados cenários que representam suas funcionalidades de colaboração entre equipes médicas, Gerenciamento de Atividades e Ciência de Situação. A comunidade científica tem empregado cenários para avaliação de aplicações ubíquas e cientes de contexto (Damasceno Vianna, H., \& Barbosa, J. L. V. 2014).

Esta seção apresenta uma implementação e avaliação das tecnologias utilizadas para a realização e concepção do Doctor Collab em uma ambiente real. Para esta implementação, foram utilizados cenários que representam suas funcionalidades. A comunidade científica tem empregado cenários para avaliação de aplicações ubíquas e cientes de contexto [Satyanarayanan 2010; Lopes et al. 2014; Vianna et al. 2014].

A comunicação do sistema se deu através de rede Wifi e os servidores Linux. Para esse estudo de caso, foram desenvolvidos duas aplicações cliente: a primeira tem por objetivo abarcar as plataformas da web baseada em Java Plataform Enterprise Edition (JavaEE) e a segunda é relacionada às plataformas de dispositivos móveis (desenvolvida para Android). Com a plataforma web é possível ter uma junção de uma série de informações como coleta de dados sensoriais, colaborações em prontuários médicos e ainda o acesso a histórico de atividades realizadas na emergência médica. 
Na Figura 5 apresenta-se a tela inicial da aplicação em que se pode acessar todas as informações do paciente. Nela, é possível ter acesso aos dados do paciente e verificar se todos os sensores estão funcionando de maneira correta. Caso o paciente necessite de algum cuidado especial que influencie diretamente no seu bem estar, isso poderá ser inserido na aplicação. A dieta restrita também poderá ser cadastrada e acompanhada dentro do Doctor Collab, além dos medicamentos utilizados durante o período de monitoramento. Essas informações poderão ser relacionadas de forma a entender em tempo real o impacto de uma medicação em um paciente.

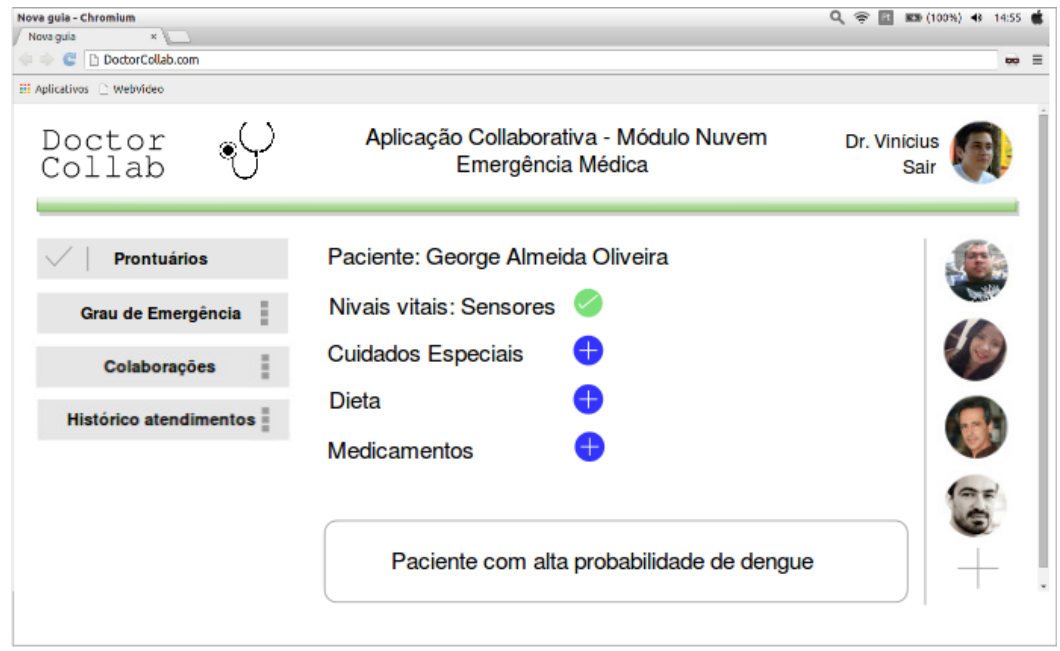

Figura 5 - Tela inicial Doctor Collab - módulo Web

Outro componente do módulo web é o acesso a inferências feitas através do cruzamento de informações e de técnicas de Redes Bayesianas. Os médicos cadastrados no sistema poderão ter acesso em tempo real ao prontuário médico de cada paciente e colaborar com a equipe responsável. Isso facilitará para que a troca de informações entre esses médicos ocorra de forma mais transparente. Todas as consultas e evoluções do quadro clínico podem ser vistas através do menus "histórico de atendimento".

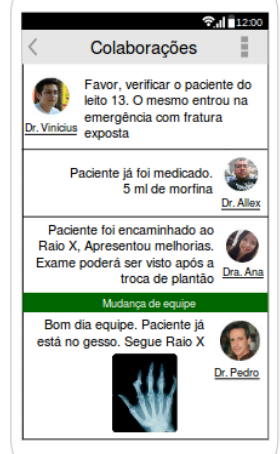

Figura 6 - Tela de Colaboração Doctor Collab - Módelo Móvel

O módulo destinado aos dispositivos móveis apresenta uma visão mais simplificada devido ao menor tamanho das telas e ainda ao dinamismo em que a aplicação será utilizada. A Figura 6 demostra a tela de colaborações. A partir dela é possível que médicos realizem conversas e prescrições formais agilizando assim a troca de informações entre as equipes. No exemplo da figura, os médicos estão dialogando sobre um paciente específico, a medicação mais adequada para a situação e caso haja a 
necessidade de um exame, o mesmo poderá ser inserido na aplicação através de uma fotografia.

Outra importante característica do modo de colaboração diz respeito ao acesso dessas informações pela equipe médica que assume o próximo plantão. Tudo poderá ser acessado de forma rápida e transparente pela nova equipe, mesmo antes do seu turno de trabalho. Os médicos poderão já se preparar para o início do trabalho, utilizando o módulo de colaboração. No Doctor Collab também estará disponível o suporte a colaborações externas, quando necessário.

\section{Trabalhos Relacionados}

A discussão dos trabalhos relacionados abrange os estudos que envolvem computação Baseada em Atividade, colaboração, ciência de situação e gerenciamento de emergência. Considerando os trabalhos apresentados nesta sessão, todos focam na colaboração entre equipes e fornecem algum tipo de implementação de comunicação síncrona ou assíncrona. Entretanto, o Doctor Collab é o único que oferece uma arquitetura em nuvem através da utilização de dispositivos móveis para estas colaborações. Quatro trabalhos utilizam contexto, porém apenas o Doctor Collab correlaciona essas informações de contexto às atividades presentes no ambiente de estudo, enriquecendo ainda mais a inferência médica.

O Estudo Activy-Based Computing [Bardram, E. 2005] apresenta uma visão sólida sobre o estudo de Computação Baseada em Atividade (activity-based computing) e a forma como o conceito coopera com as atividades humanas. O Framework em estudo apresentada: i) Suporte a gerência de tarefas humanas utilizando computador; ii) suporte a mobilidade e distribuição de atividades através de ambientes heterogêneos; iii) Suporte a colaboração assíncrona, autorizando desta maneira que diversas outras pessoas possam participar; e iv) suporte a colaboração síncrona, criando assim ambientes de colaboração em tempo real, a exemplo de video-conferências.

$\mathrm{O}$ artigo Community-based collaborative information system for emergency management [Juan Li et al. 2012] buscou abordar como se pode trabalhar com um sistema colaborativo baseado em comunidade para locais classificados como emergenciais, a exemplo de tsunamis, enchentes e epidemias. Nessas circunstâncias é muito importante se trabalhar com um sistema bem organizado, por existir um alto nível de stress e qualquer falha de organização da informação poderá acarretar em grandes problemas. Para isso, o artigo cita o uso da técnica de tomada de decisão em multi-critérios (Multi-criteria decision making-MCDM), importante aliado para esse tipo de processo. Essa técnica pode ajudar as pessoas que utilizam a plataforma em busca dos seguintes tópicos: i) avaliar a situação atual; ii) encontrar soluções satisfatórias e iii) ter respostas apropriadas em um tempo hábil.

Outro interessante estudo é o de nome ReticularSpaces [Bardram, J. et al. 2012] que apresenta uma análise de espaços inteligentes através do uso Computação baseada em Atividade com suporte a colaboração utilizando dispositivos móveis. O trabalho tem como foco o suporte a: i) unificar interação entre aplicações através do uso do ReticUI; ii) O gerenciamento de tarefas complexas entre usuários e displays, iii) Utilização remota ou local dos serviços disponibilizados; iv) Colaboração tanto entre usuários locais quanto usuários remotos. Utiliza-se a computação baseada em atividade para formalizar a informação trafegada dentro da aplicação através do uso de ontologia.

O estudo A Mobile Collaborative Approach To Improve Healthcare Tasks Planning [Zidani, Chorfi, Lezzar. 2014] apresenta uma análise do uso de dispositivos 
móveis colaborativos em prol do melhoramento do planejamento de tarefas focadas à saúde. A plataforma foi desenvolvida em sistema Android por oferecer a estrutura necessária para a execução dos experimentos de colaboração utilizando dispositivos móveis. Para isso, foi desenvolvido um agrupamento síncrono de dispositivos móveis Android no intuito de habilitar uma colaboração em tempo real onde membros desses grupos podem estar separados geograficamente mas ainda assim, conseguirem utilizar o sistema. Esse trabalho utilizou a Teoria de Utilitários de múltiplos atributos para ajudar na comparação com outros diferentes planejamentos de Health Insurance, uma técnica importante para análise de decisões. O modelo construído possibilita o uso de dados heterogêneos. Para o planejamento das informações de cada dado foi utilizado a estrutura em nuvem do DaaS (Dados como Serviço). O framework foi implementado como SaaS (Software como serviço) para a customização das informações que foram categorizadas em: i) Registros de seguro; ii) prescrições de remédios utilizados pelos pacientes; e iii) respostas ao paciente. A arquitetura do sistema com foco à saúde baseado em computação em nuvem possui algumas entidades como: i) Healthcare providers a exemplo de Hospitais, Clinicas médicas, farmácias e laboratórios; e ii) Health insurance providers.

Por fim, o estudo $A$ cloud based health insurance plan recommendation system [Abbas et al. 2015] utilizou a Teoria de Utilitários de múltiplos atributos para ajudar na comparação com outros diferentes planejamentos de Health Insurance, uma técnica importante para análise de decisões. O modelo construído possibilita o uso de dados heterogêneos. Para o planejamento das informações de cada dado foi utilizado a estrutura em nuvem do DaaS (Dados como Serviço). O framework foi implementado como SaaS (Software como serviço) para a customização das informações que foram categorizadas em: i) Registros de $s$ eguro; ii) prescrições de remédios utilizados pelos pacientes; e iii) respostas ao paciente.

\section{Conclusão}

A concepção de um modelo em nuvem para apoiar a colaboração e troca de informações entre equipes médicas, de forma a utilizar a ciência de situação e computação baseada em atividade, se mostra uma solução oportuna para a avaliação de um forma mais rápida, transparente e confiável entre ambientes de emergências, assim como estimula a colaboração entre médicos especialistas e padronização de informações.

Desta forma, através do incentivo a essas colaborações, o médico estará de forma mais rápida com o acesso a dados e inferências que melhorarão seu diagnóstico. O estado clínico poderá ser acompanhado em tempo real, até mesmo pelo próprio paciente. $\mathrm{O}$ uso de estruturas que estimulem a computação móvel enriquece o dinamismo da aplicação. Neste ponto, o médico poderá receber da aplicação inferências a respeito de, por exemplo, como um paciente se comportou nas últimas horas depois de uma determinada medicação.

Como principal contribuição científica, a proposta de um modelo colaborativo em nuvem móvel utilizando áreas com Computação Baseada em Atividade e Ciência de Contexto através de Redes Bayesianas e técnicas de inferência computacional NP-Hard mais Ontologia. Como resultado da avalição do modelo, espera-se criar uma solução satisfatória de uso real e aplicada as necessidade de uma emergência médica.

Em se tratando da continuidade de pesquisa, alguns aspectos deverão ser considerados em trabalhos futuros: (i) realizar uma avaliação de desempenho da 
aplicação Doctor Collab Web e Doctor Collab Móvel, verificando como o modelo se comporta em uma situação cotidiana; (ii) Verificar como os mecanismos de inferência médica podem ser aplicados em diferentes casos reais de uso; (iii) aprimorar os estudos em prol da segurança do modelo em nuvem privada e (iv) expandir os mecanismos de interação entre equipes médicas.

\section{Agradecimentos}

Os autores gostariam de agradecer ao CNPq pelo apoio a essa pesquisa.

\section{Referências}

Abbas, A., Bilal, K., Zhang, L., \& Khan, S. U. (2015). A cloud based health insurance plan recommendation system: A user centered approach. Future Generation Computer Systems, 43, 99-109.

Bardram, E. (2005). Activity-based computing: support for mobility and collaboration in ubiquitous computing. Personal and Ubiquitous Computing,9(5), 312-322.

Bardram, J., Gueddana, S., Houben, S., \& Nielsen, S. (2012). ReticularSpaces: activitybased computing support for physically distributed and collaborative smart spaces. In Proc. SIGCHI Human Factors in Computing Systems (pp. 2845-2854). ACM.

Damasceno Vianna, H., \& Barbosa, J. L. V. (2014). A model for ubiquitous care of noncommunicable diseases. Biomedical and Health Informatics, 18(5), 1597-1606.

Dinh, H. T., Lee, C., Niyato, D., \& Wang, P. (2013). A survey of mobile cloud computing: architecture, applications, and approaches. Wireless communications and mobile computing, 13(18), 1587-1611.

Endsley, M. R. (1995). Toward a theory of situation awareness in dynamic systems. Human Factors: J. Human Factors and Ergonomics Society, 37(1), 32-64.

Fernando, N., Loke, S. W., \& Rahayu, W. (2013). Mobile cloud computing: A survey. Future Generation Computer Systems, 29(1), 84-106.

Fouzi Lezzar, F. L., Abdelmadjid Zidani, A. Z., \& Atef Chorfi, A. C. (2014). Using A Mobile Collaborative Approach To Improve Healthcare Tasks Planning. Int. Journal of Multimedia and Ubiquitous Engineering,8(3), 407-420.

Juan Li, Qingrui Li, Chao Liu, Samee Ullah Khan, Nasir Ghani. Community-based collaborative information system for emergency management. Computer Operation Res. 42 (2012)

Lopes, J., Souza, R., Gadotti, G., Pernas, A., Yamin, A., \& Geyer, C. (2014). An Architectural Model for Situation Awareness in Ubiquitous Computing.Latin America Transactions, IEEE (Revista IEEE America Latina), 12(6), 1113-1119.

Musen, M. A., Middleton, B., \& Greenes, R. A. (2014). Clinical decision-support systems. In Biomedical informatics (pp. 643-674). Springer London.Noy, N. F., \& McGuinness, D. L. (2001). Ontology development 101: A guide to creating your first ontology.

Shelbourn, M. A., Bouchlaghem, D., Anumba, C., \& Carrillo, P. (2006). A decision making framework for planning and implementing collaborative working. In Proc. Joint International Conference on Computing and Decision Making in Civil and Building Engineering, Montreal, Canada, June 2006 (pp. 930-944).

Vianna, H. D., Barbosa, J. (2014) A Model for Ubiquitous Care of Noncommunicable Diseases. IEEE J Biomed Health, 18, 1597-1606.

Weiser, M. (1991). The computer for the 21st century. Scientific American,265(3), 94104. 